\title{
Redes de Apoio à Mulher em Situação de Violência durante a Pandemia de Covid-19
}

\section{Support Networks for Women in Situations of Violence during the Covid-19 Pandemic \\ Redes de Apoyo a Mujeres en Situación de Violencia durante la Pandemia de Covid-19}

\author{
Luciana Barbalho Pontes ${ }^{1}$ \\ Universidade do Estado do Pará (UEPA) \\ Maria Beatriz Reis Dionísio \\ Maria Alice Centanin Bertho \\ Viviane Dutra Gama \\ Sabrina Mazo D’Affonseca \\ Universidade Federal de São Carlos (UFSCar)
}

\begin{abstract}
Resumo
A adoção de medidas de distanciamento social decorrentes da pandemia de covid-19 agravou o fenômeno da violência contra as mulheres, especialmente a praticada por parceiros íntimos (VPI). O presente artigo tem por objetivo analisar a VPI em contexto de pandemia e descrever iniciativas de proteção à mulher em situação de VPI. A partir da análise sobre a VPI, suas consequências e formas de enfrentamento, foram descritas ações tanto das redes formais quanto das redes informais de apoio à mulher. Por meio de buscas no Google e nas redes sociais, foram levantadas as seguintes estratégias de combate à VPI: a capacitação dos profissionais de saúde para identificação de sinais de violência e o desenvolvimento de aplicativos, projetos e campanhas de conscientização, incentivo à denúncia e acolhimento das vítimas. É notória a necessidade das redes de apoio para a prevenção da VPI e de pesquisas futuras que avaliem as potencialidades dessas estratégias.

Palavras-chave: violência por parceiro íntimo, mulheres, pandemias, infecções por coronavírus, apoio social
\end{abstract}

\begin{abstract}
The adoption of social distance measures resulting from the covid-19 pandemic has aggravated the phenomenon of violence against women, especially that practiced by an intimate partner (IPV). This article aims to reflect on IPV in a pandemic context and describe existing initiatives to protect women in a situation of IPV. Based on the analysis of IPV, its consequences and coping strategies, actions were described both from formal and informal networks to support women. Searches on Google and social media raised the following strategies for combating IPV: the training of health professionals to identify signs of violence and the development of apps, projects and awareness-raising campaigns, to report violence and welcome victims in a pandemic context. There is a clear need for support networks to prevent the IPV and for future research to assess the potential of these strategies.

Keywords: intimate partner violence, women, pandemics, coronavirus infections, social support
\end{abstract}

\section{Resumen}

La adopción de medidas de distancia social derivadas de la pandemia de covid-19 ha agravado el fenómeno de la violencia contra las mujeres, especialmente la practicada por parejas (VPM). Este artículo tiene como objetivo analizar la VPM en contexto de pandemia y describir iniciativas para proteger a las mujeres en esa situación. A partir del análisis de la VPM, sus consecuencias y formas de afrontamiento, se describieron acciones tanto de las redes formales como informales de apoyo a las mujeres. A través de búsquedas en Google y redes sociales, se plantean las siguientes estrategias para combatir la VPM: la capacitación de profesionales de la salud para identificación de señales de violencia y el desarrollo de aplicaciones, proyectos y campañas de sensibilización, incentivo a la denuncia y acogida de las víctimas. Las redes de apoyo resultan esenciales para la prevención de VPM. Futuras investigaciones podrán evaluar el potencial de estas estrategias.

Palabras clave: violencia de pareja, mujeres, pandemias, infecciones por coronavirus, apoyo social

\footnotetext{
${ }^{1}$ Endereço para contato: Rodovia Washington Luis, km 235, Laboratório de Análise e Prevenção da Violência da Universidade Federal de São Carlos (LAPREV/UFSCar), departamento de Psicologia, São Carlos, São Paulo. Telefone: (16) 3351-8745. E-mail: lu_barbalho@yahoo.com.br
} 


\section{Introdução}

O ano de 2020 foi marcado pelo que a Organização das Nações Unidas (ONU) reconheceu como uma pandemia sem precedentes (ONU, 2020). Até o momento, a covid-19, doença causada pelo novo coronavírus SARS-CoV-2, já dizimou centenas de milhares de vidas ao redor do globo (Johns Hopkins University, 2020). Com o objetivo de suprimir a transmissão do vírus, governos de todo o mundo passaram a coordenar medidas de isolamento e distanciamento social da população, resultando em milhões de pessoas confinadas no ambiente doméstico, com a convivência social restrita. Apesar da necessidade das medidas no combate à pandemia, estas escancaram as desigualdades de gênero e agravam a exposição das mulheres ao risco de violência entre parceiros íntimos (VPI). A VPI vem sendo considerada a forma mais comum de violência contra a mulher, excedendo a prevalência de todas as outras formas de abuso físico e sexual na vida das mulheres (Heise, 2011; World Health Organization [WHO], 2014; 2016).

O presente trabalho tem como principais objetivos analisar a VPI em contexto de pandemia e descrever iniciativas de proteção à mulher em situação de VPI. Como objetivos específicos, buscamos descrever e discutir estratégias relacionadas ao funcionamento das redes formais de proteção à mulher e realizar um mapeamento de ações de redes informais de proteção à mulher em situação de VPI. Para tal, foram realizadas buscas no Google e nas redes sociais, a fim de levantar as estratégias de combate à VPI em ação, especialmente no contexto da pandemia. A partir deste trabalho, espera-se contribuir para a divulgação de tais redes e ampliar seu alcance por parte das mulheres em situação de VPI, dos profissionais que atuem com esse público e da população em geral.

\section{A Violência entre Parceiros Íntimos em Contexto de Pandemia}

A VPI refere-se a qualquer comportamento dentro de um relacionamento íntimo que cause problemas físicos, danos psicológicos ou sexuais àqueles na relação. Esse comportamento inclui: atos de agressão física (tapas, socos, chutes etc.), abuso psicológico (intimidação, depreciação, humilhação constante), abuso sexual (relações sexuais forçadas e coerção sexual) e comportamentos de controle do parceiro (isolar uma pessoa de sua família e amigos, monitorar seus movimentos, exercer controle financeiro e restringir o acesso a informações ou assistência) (Krug, Mercy, Dahlberg, \& Zwi, 2002). A VPI não deve ser compreendida a partir de uma análise simplista, mas sim como um fenômeno multifacetado, baseado na interação entre aspectos pessoais, situacionais e fatores socioculturais (Heise, 1998; 2011). Como destaca a a Entidade das Nações Unidas para a Igualdade de Gênero e o Empoderamento das Mulheres [ONU Mulheres] (2020), o período de isolamento pode representar um fator de risco às ocorrências de VPI, devido às dificuldades econômicas acentuadas pela pandemia, filhos em tempo integral em casa, acúmulo de funções e conciliação de demandas familiares e profissionais no mesmo espaço físico, os quais podem acarretar conflitos entre os parceiros.

Alguns impactos do cenário atual sobre os casos de violência já podem ser identificados. Segundo a nota técnica emitida pelo Fórum Brasileiro de Segurança Pública [FBSP] (2020), o número de ocorrências atendidas pela Polícia Militar (PM) relacionadas à violência doméstica no Estado de São Paulo cresceu 44,9\% no mês de março de 2020, em relação ao mesmo 
mês do ano anterior. No Estado do Rio de Janeiro, o Instituto de Segurança Pública [ISJ] (2021) publicou um "Monitor da Violência Doméstica e Familiar contra a Mulher no Período de Isolamento Social", indicando uma queda no número de Registros de Ocorrências na Polícia Civil (presenciais ou on-line) no período de 13 de março a 31 de dezembro de 2020, quando comparado com o mesmo período em 2019. Ainda foi identificado que o número de ligações para o Disque Denúncia sobre "violência contra a mulher" reduziu em 20,3\%. Por outro lado, o Serviço 190 da PM apresentou um aumento de 1,6\% na quantidade de ligações referentes a "Crimes contra a mulher". Já o serviço do Ligue 180, que recebe denúncias de violência contra a mulher, registrou 7.714 ligações em março de 2020, comparadas a 8.440 ligações recebidas no mesmo período em 2019 (redução de 8,6\%).

O paradoxo entre o aumento de ocorrências atendidas pela PM no Estado de São Paulo, por exemplo, e o decréscimo do número de denúncias no território nacional e no Estado do Rio de Janeiro deve ser compreendido de maneira crítica. As mulheres vítimas de VPI estão confinadas sob supervisão constante do seu agressor, o que impossibilita seu acesso aos canais de denúncia, resultando na redução dos números (FBSP, 2020). O aumento do número de casos de VPI durante a pandemia é, portanto, uma intensificação da realidade cotidiana dessas mulheres (ONU Mulheres, 2020; Peterman et al., 2020).

Além disso, nesse período, o acesso das mulheres aos serviços, especialmente presenciais, de apoio às vítimas, tais como de assistência social, saúde, segurança pública e justiça, instituições e mecanismos que compõem a rede formal de proteção, também fica prejudicado (Vieira, Garcia, \& Maciel, 2020). Apesar de o contexto da pandemia agravar o isolamento e as dificuldades de denúncia, estas não são inéditas para aquelas que vivenciam esse contexto de violência, em que uma privação de liberdade e autonomia já é experienciada, ainda que de outras formas (Albuquerque Netto, Moura, Queiroz, Leite, \& Silva, 2017). Além disso, ao procurarem apoio para lidar com a situação vivenciada, nem sempre as mulheres o conseguem da maneira esperada, pois o caminho percorrido ao acesso dos serviços especializados é difícil e cheio de obstáculos (D’Oliveira \& Schraiber, 2013; Sagot, 2000).

Nesse sentido, as redes de apoio informais apresentam um papel importante no auxílio às mulheres em situação de VPI. De acordo com D'Oliveira e Schraiber (2013), estudos demonstraram que essas mulheres buscam ativamente superar a situação vivenciada, de modo a interromper a violência, seja conversando com alguém, seja pedindo ajuda. Em um estudo realizado por Gregory, Taylor, Pitt, Feder e Williamson (2019), os autores identificaram que a vasta maioria das mulheres vítimas de violência escolhe revelar e solicitar apoio de amigos ou familiares em quem confiam em vez de procurarem serviços especializados. Estatísticas de crimes na Inglaterra e no País de Gales indicam que, de $88 \%$ das vítimas que relataram suas experiências de VPI em 2016, $80 \%$ haviam revelado o caso para sua rede informal, enquanto somente $43 \%$ procuraram ajuda profissional (Office for National Statistics [ONS], 2016). Apesar de haver grande variabilidade na efetividade do suporte informal, a maior parte das vítimas consegue identificar pelo menos uma pessoa da sua rede capaz de the fornecer suporte de alguma forma (Gregory et al., 2019). Assim, essa rede de apoio informal representa um importante fator de proteção e pode ser a chave para a ativação da rede de apoio formal em casos de VPI, especialmente em tempos de isolamento social.

Contudo, deve-se salientar que o cenário ideal deveria priorizar os acessos formais a serviços da rede de enfrentamento à violência, ampliando possibilidades oficiais de 
proteção àquelas mulheres que estão em situação de VPI e, com isso, garantindo o empoderamento delas. Cumpre destacar que, desde a Plataforma de Ação de Pequim, preconiza-se a importância de que a mulher adquira o controle sobre o seu desenvolvimento, devendo o governo e a sociedade criar as condições para tal, além de apoiá-la nesse processo (Frossard, 2006).

Conforme apontado por Vieira et al. (2020), medidas para fortalecer o combate à VPI em tempos de covid-19 devem ser amplamente fomentadas, tais como o aumento das equipes nas linhas diretas de prevenção e combate à violência, a ampla divulgação dos serviços disponíveis, a capacitação dos trabalhadores da saúde para identificação de fatores de risco, a expansão e o fortalecimento das redes de apoio às mulheres, incluindo aumento no número de vagas nos abrigos e o encorajamento das redes virtuais de suporte social. Dutra, Prates, Nakamura e Villela (2013, p. 1302) já haviam apontado para a necessidade de ". . incorporar novos modos de fazer e de tecer a rede de apoio às mulheres, possibilitando um fluxo entre os serviços, instituições e atores significativos no entorno das mulheres".

Considerando o contexto atual, visando ao combate à VPI, ações que tornem as redes de apoio visíveis às mulheres e à sociedade têm sido trazidas à tona. Para isso, diferentes instituições e organizações estão usando os meios de comunicação para levarem informações sobre violência, abrirem caminhos ao fortalecimento de suas redes de apoio informais e à construção de novas conexões que ofereçam suporte a elas e acesso à rede formal de proteção. Diante disso, as medidas atualmente fomentadas podem representar auxílios importantes para o combate à VPI, não devendo ser implementadas exclusivamente durante a pandemia, e sim mantidas permanentemente ativas. Ressalta-se ainda que o enfrentamento à VPI nesse contexto não pode limitar-se apenas ao acolhimento das denúncias (Vieira et al., 2020), visto que a promoção do suporte para conectar as mulheres com outros serviços de que precisam é essencial no enfrentamento da VPI (Baragatti, Carlos, Leitão, Ferriani, \& Silva, 2018).

\section{A Rede de Apoio Formal}

\section{O Papel do Poder Público no Enfrentamento da Violência contra a Mulher}

O Brasil é signatário de todos os acordos internacionais que asseguram os direitos das mulheres e a eliminação de todas as formas de discriminação e violência baseadas no gênero. Dentre eles, destaca-se a Declaração e Plataforma de Ação da IV Conferência das Nações Unidas sobre a Mulher, realizada em Pequim em 1995 e considerada o principal marco mundial para a proteção e promoção dos direitos das mulheres (Frossard, 2006). Seu principal legado foi um conjunto de objetivos estratégicos traçados, como “. . . um guia abrangente para orientar governos e sociedade no aperfeiçoamento do marco legal, na formulação de políticas públicas e na implementação de programas para promover a igualdade e para evitar a discriminação", tendo como inovações o conceito de gênero, a noção de empoderamento e o enfoque da transversalidade (Frossard, 2006, p. 149).

Como consequência da sua participação ativa na Conferência de Pequim, o Brasil beneficiou-se de um diálogo e interação construtivos entre o governo e a sociedade civil, com forte articulação do movimento de mulheres, para a formulação de políticas públicas que incorporassem a perspectiva de gênero de maneira transversal (Frossard, 2006). A partir de então, o governo brasileiro criou a Secretaria de Políticas para as Mulheres, em 2003, 
a qual foi responsável, em 2007, por desenvolver o Pacto Nacional pelo Enfrentamento à Violência Contra a Mulher. Este consiste em um acordo entre o governo federal, os governos dos estados e dos municípios brasileiros para o planejamento de ações que consolidassem a Política Nacional pelo Enfrentamento à Violência contra as Mulheres por meio da implementação de políticas públicas integradas em todo o território nacional. Foi o responsável pela criação de uma rede especializada de serviços para mulheres em situação de violência, constituída por Centros de Atendimento à Mulher em situação de violência; Casas de Abrigo; Casas de Acolhimento Provisório; Delegacias Especializadas de Atendimento à Mulher; núcleos especializados de atendimento às mulheres nas delegacias comuns; Núcleos da Mulher nas Defensorias Públicas; Promotorias Especializadas; Juizados Especiais de Violência Doméstica e Familiar contra a Mulher; Central de Atendimento à Mulher - Ligue 180; Ouvidoria da Mulher; serviços de saúde voltados para o atendimento aos casos de violência sexual e doméstica; e Serviços de Atendimento em Fronteiras Secas (Brasil, 2011).

Também como consequência de sua participação na Conferência de Pequim, o Brasil aprovou a Lei Maria da Penha (Brasil, 2006), a qual foi o principal marco legal no enfrentamento à violência doméstica e familiar contra a mulher, estabelecendo medidas de assistência e proteção às mulheres vítimas de violência por meio de um conjunto articulado de ações da União, do Estado e do Distrito Federal, dos Municípios e organizações não governamentais. Contudo, a aplicação da Lei Maria da Penha depende em grande parte da oferta de serviços e articulação da rede de proteção às mulheres.

Apesar do crescimento do número de serviços ao longo dos anos, a maioria ainda se encontra em capitais e regiões metropolitanas e, em muitos estados nos quais os serviços se encontram disponíveis, estes não conversam entre si e nem sequer poderiam ser chamados de "rede". Tal realidade dificulta o acesso das mulheres ao serviço e, consequentemente, a implementação da Lei Maria da Penha (Campos, 2015).

A situação se agrava ainda mais em tempos de distanciamento social. Por isso, considerando as dificuldades de acesso aos canais de denúncia formais durante a pandemia de covid-19, o Ministério da Mulher, Família e Direitos Humanos (MMFDH) adotou o uso de plataformas digitais nos canais de atendimento da Ouvidoria Nacional dos Direitos Humanos (ONDH) como forma de contornar tais dificuldades e promover o acolhimento das denúncias de violência contra a mulher (Vieira et al., 2020). Foram disponibilizados o aplicativo Direitos Humanos BR e o site da ONDH (https://ouvidoria.mdh.gov.br), a partir do qual se pode acessar o Disque 100 e o Ligue 180. Por meio desses canais, não somente as vítimas, mas familiares, vizinhos, ou mesmo desconhecidos podem enviar, de forma anônima, fotos, vídeos, áudios ou outros que registrem situações de violência doméstica e outras violações de direitos humanos.

Outra iniciativa, criada em junho de 2020 pelo Conselho Nacional de Justiça (CNJ) e pela Associação dos Magistrados Brasileiros (AMB), sendo aderida pelo governo federal por meio do MMFDH, é a campanha "Sinal Vermelho contra a violência doméstica". A campanha incentiva mulheres vítimas de violência a desenharem um " $X$ " na mão e exibi-la ao atendente nas mais de 10 mil farmácias que aderiram à campanha. Uma cartilha e tutorial foram oferecidos às farmácias para capacitação dos funcionários quanto ao acolhimento das vítimas e acionamento da Central de Atendimento à Mulher - Ligue 180 e das autoridades competentes (https://www.amb.com.br/sinalvermelho/). 
Ainda, a Defensoria Pública do Estado de São Paulo elaborou um guia sobre os direitos das mulheres e a covid-19, o qual contém informações para identificação de violência doméstica e familiar, violência sexual e obstétrica, além de algumas ações a serem tomadas em casos de violência, como acionar medidas protetivas por meio de Defensoria Pública / ou advogado(a), Ministério Público ou Delegacias de Polícia. Ademais, fornece dados como o contato desses órgãos e aconselha quanto à importância de ter em mãos, durante a denúncia, provas que auxiliem a demonstrar a violência sofrida, como mensagens de texto, áudios, fotografias e outros (Defensoria Pública do Estado de São Paulo, 2020).

Em tempos de pandemia, além de o poder público desempenhar um papel essencial ao instrumentalizar as possibilidades de denúncia da VPI, os profissionais de saúde, por atuarem em um serviço essencial, também exercem um papel extremamente relevante para a revelação de casos de VPI, sendo, frequentemente, o único suporte para muitas mulheres em situação de violência, pois terão a chance de detectar sinais de violência e encaminhar a suspeita.

\section{O Papel dos Profissionais de Saúde no Enfrentamento da Violência contra a Mulher}

Muitos profissionais dos serviços de saúde têm dificuldade de lidar com os casos de violência que chegam para atendimento, seja por não o reconhecerem como um problema da área de saúde, por lidarem mal com as queixas difusas e imprecisas, as quais nem sempre correspondem a uma patologia específica, ou por sequer reconhecerem a situação como um problema que requer uma intervenção, seja pela ausência desse tema na formação inicial e continuada dos profissionais; pelo sentimento de desresponsabilização dos profissionais pelo problema, enxergando qualquer ação nesse sentido como iniciativa pessoal, e não como função profissional; pela insegurança dos trabalhadores; pela falta de protocolos específicos de atendimento e falta de conhecimento sobre a rede de serviços, ou mesmo por concepções pessoais equivocadas a respeito do fenômeno, contribuindo, assim, para a invisibilidade, perpetuação e agravamento da violência (Almeida, Silva, \& Machado, 2013; Cavalcanti et al., 2020; D’Oliveira \& Schraiber, 2013; Garbin, Garbin, Dossi, \& Dossi, 2006; Kind et al., 2013; Osis, Duarte, \& Faúndes, 2012; Sinclair, 2019).

Anteriormente, os serviços de atendimento às mulheres não possuíam sistemas adequados de identificação e registro da violência, o que levava a diagnósticos, atividades de prevenção e modelos de atendimento inadequados para responder às necessidades das muIheres afetadas, resultando na invisibilidade e subnotificação do fenômeno (Sagot, 2000). A promulgação da Lei n. 10.778 (Brasil, 2003), que institui a notificação compulsória de violência contra a mulher nos serviços de saúde, contribuiu para a visibilização do fenômeno.

Entre os anos de 2009 e 2017, foram registrados 208.582 casos de violência contra a mulher perpetrada por seus parceiros íntimos no Sistema de Informação de Agravos de Notificação (SINAN). Este número equivale a 13,5\% de todas as notificações registradas na categoria "violência doméstica, sexual e/ou outras violências" atendidas pelo Sistema Único de Saúde (SUS) neste período (Departamento de Informática do Sistema Único de Saúde 2019). Tais dados indicam a necessidade de capacitação dos profissionais da saúde para reconhecer e intervir em situações de VPI, a qual se torna ainda mais latente em tempos de distanciamento social. 
Segundo García-Moreno et al. (2014), os profissionais de saúde devem estar capacitados para identificar, oferecer suporte inicial após a revelação ou identificação, oferecer cuidados clínicos, realizar follow-up e encaminhar os casos de violência contra a mulher. No entanto, verifica-se que o obstáculo mais evidente para a identificação desses casos refere-se ao desconhecimento das características e das consequências físicas e psicológicas da VPI (Hasse \& Vieira, 2014). Uma vez que há a revelação ou identificação de uma situação de violência, é vital que a mulher receba suporte adequado do profissional de saúde (García-Moreno et al., 2014), com uma primeira escuta empática, que valide a experiência da mulher e ofereça suporte condizente com seus desejos e necessidades.

Schraiber e D'Oliveira (2002) descrevem um serviço de atendimento a mulheres vitimizadas (Conflitos Familiares Difíceis [CONFAD]) e detalham de maneira clara como responsabilidade do profissional: 1) ouvir a história com atenção; 2) tentar entender o problema; 3) procurar redes de apoio já existentes (amigos, família); 4) identificar riscos como ameaças ou presença de armas e dar conselhos que colaborem para que a vítima permaneça segura; 5) discutir sonhos e expectativas que auxiliem a mulher a identificar meios de mudança, 6) dar suporte às escolhas e ao processo da mulher; 7) clarificar que o fenômeno da violência de gênero é de natureza social e que há muitos casos similares ao que ela está passando; 8) saber respeitar opiniões e limites impostos pela mulher sem julgá-la.

O momento do encaminhamento é crucial no suporte oferecido às vítimas. No entanto, vale ressaltar que a avaliação e identificação em larga escala de casos de violência em um contexto marcado pela escassez de serviços traz poucos benefícios à mulher e sobrecarrega o profissional de saúde (García-Moreno et al., 2014). Emerge daí a necessidade de estabelecimento, divulgação e integração de serviços de proteção. Para mais informações sobre capacitação de profissionais de saúde, vide cartilha elaborada por Soares (2005).

Mesmo em período da pandemia, os elementos anteriormente descritos devem manter-se como norteadores da prática profissional. Ainda que o profissional esteja sob extrema pressão, é seu dever comprometer-se com a avaliação e atenção exigidas pelas situações de violência (WHO, 2020). Segundo orientações divulgadas pela Organização acerca da particularidade do momento atual, caso não seja possível interação presencial, os profissionais devem buscar recursos (e.g., tecnológicos) para comunicação com as vítimas, sempre visando à segurança delas. Destaca-se a orientação de que uma mulher vítima de violência que esteja com suspeita ou diagnóstico confirmado de covid-19 deve ser tratada da mesma maneira que outras vítimas. O profissional deve assegurar a sua própria proteção sem, entretanto, perder de vista os cuidados necessários com a paciente. A Organização Mundial da Saúde (OMS) recomenda ainda que gestores de unidades de saúde: ajudem na identificação, organização e integração de serviços de auxílio a vítimas e tornem essa lista disponível para usuários e profissionais de saúde; deem suporte aos profissionais no cuidado às vítimas; preparem-se para prestar auxílio rápido, criando planos de resposta efetivos; e informem as vítimas sobre como proteger-se, fornecendo cartões contendo números de serviços relevantes para as mulheres (WHO, 2020).

\section{As Redes Informais: Mobilizações da Sociedade para a Denúncia}

Além das redes formais de proteção à mulher em situação de VPI, as redes informais têm se mostrado um suporte importante para essas mulheres. Para os propósitos do presente 
artigo, utiliza-se a denominação "redes informais" para fazer alusão às redes sociais ou redes de apoio da mulher, baseadas em relações de parentesco ou amizade, vínculo pessoal, intimidade e/ou socialização (Dutra et al., 2013).

É comum que vítimas de VPI passem por um estado de individualização e se afastem das suas redes de apoio informais, quer seja por temerem as ameaças recebidas ou vergonha de se manterem na relação, refletindo em optarem por não partilhar o que estão passando (Sinclair, 2019). Se, por um lado, sua rede de apoio está fragilizada, por outro, há um aumento de poder do parceiro, culminando no distanciamento da mulher dos suportes especializados para auxiliar no processo de rompimento do silêncio da violência (Dutra et al., 2013). Assim, como forma de as mulheres conhecerem seus direitos ou serviços de assistência disponíveis e terem acesso facilitado a canais de denúncia, organizações não governamentais, coletivos e empresas que adotaram a prevenção da violência contra a mulher como uma área de ação social têm se mobilizado nos últimos anos, especialmente no contexto de pandemia, resultando no desenvolvimento de aplicativos, cartilhas e inclusive inteligências artificiais que contribuem para a denúncia das situações de violência e acolhimento das vítimas.

Novos mecanismos de apoio às vítimas usando a tecnologia surgiram e outros já existentes cresceram ainda mais durante a pandemia. O Projeto Justiceiras, por exemplo, tomou a iniciativa de reunir voluntárias de diversas áreas para oferecerem, através do aplicativo de mensagem WhatsApp, atendimento jurídico, psicológico e assistencial às vítimas de violência (Borges, 2020). O projeto realizado pelo Instituto Nelson Wilians, em parceria com o Instituto Justiça de Saia e Instituto Bem Querer Mulher, já soma mais de 1.300 voluntárias e já auxiliou mais de 376 mulheres (Instituto Nelson Wilians [INW], 2020). Para receber acolhimento ou orientações, a mulher deve preencher um formulário obtido mediante o número de WhatsApp utilizado pelo projeto e, por meio deste, o caso é encaminhado para uma voluntária especializada, a partir da demanda da vítima (Aun, 2020).

Empresas de transporte também estão fazendo parte de iniciativas de combate à violência de gênero. O aplicativo de delivery Rappi criou como ferramenta de auxílio o botão SOS Justiceiras, em parceria com o Projeto Justiceiras, em que as mulheres que estão passando por uma situação de violência podem acioná-lo e serem direcionadas a conversas via WhatsApp com as profissionais voluntárias (Cerioni, 2020). Igualmente, a empresa Uber disponibiliza, por meio de uma assistente virtual, lançada em parceria com o Instituto Avon e Wieden+Kennedy, orientações às vítimas de violência doméstica e algumas perguntas para identificar o grau de risco a que essa mulher está exposta durante seu acesso (Instituto Avon, 2020). Se for necessário recorrer a algum atendimento presencial em delegacias, hospitais ou serviços assistenciais, a Uber disponibiliza ainda um código promocional para que a muIher solicite uma viagem gratuita no aplicativo para esses locais (Uber, 2020). Ainda fazendo uso de ferramentas tecnológicas para apoio às mulheres que enfrentam violência doméstica durante o isolamento social por conta do covid-19, há o site Mapa do Acolhimento, responsável por reunir psicólogas e advogadas que fornecem apoio e orientações sobre a situação (Instituto Avon, 2020). A iniciativa existe desde 2016 e já havia realizado mais de 4.800 encaminhamentos até o fim de 2019 (Aun, 2020). Com a pandemia, o projeto ganhou destaque a partir da sua plataforma on-line por conectar mulheres com voluntárias mais próximas com disponibilidade para atendê-las (Mapa do Acolhimento, 2020). 
Por vezes, o caminho de recorrer diretamente aos dispositivos legais envolve medo e vergonha por parte da vítima, o que justifica a importância desses recursos para que mulheres em situação de violência possam ter acesso facilitado aos serviços e recebam o acolhimento necessário (Albuquerque Netto et al., 2017). Essas e outras iniciativas semelhantes estão descritas na Tabela 1 e compartilham a preocupação em, de algum modo, informar, acolher e fortalecer a mulher para a realização da denúncia da violência sofrida.

Tabela 1

Recursos Tecnológicos Disponíveis para o Enfrentamento da Violência contra a Mulher

\begin{tabular}{|c|c|}
\hline Ações & Descrição \\
\hline Projeto Justiceiras & $\begin{array}{l}\text { Grupo de psicólogas, assistentes sociais e advogadas voluntárias que } \\
\text { acolhem e orientam, através do WhatsApp, mulheres vítimas de } \\
\text { violência doméstica. }\end{array}$ \\
\hline Rappi + Projeto Justiceiras & $\begin{array}{l}\text { Através da parceria entre o Projeto Justiceiras e a empresa de entregas } \\
\text { Rappi, é possível acionar por ajuda clicando na opção "SOS Justiceiras" } \\
\text { do próprio aplicativo. }\end{array}$ \\
\hline $\begin{array}{l}\text { Assistente virtual } \\
\text { Instituto Avon, Uber, } \\
\text { Wieden+Kennedy }\end{array}$ & $\begin{array}{l}\text { Assistente virtual que pode ser contatada através de um número do } \\
\text { WhatsApp, a qual identificará, por meio de algumas perguntas, o grau } \\
\text { de risco que a mulher está correndo. Ao ser identificado o grau de risco, } \\
\text { a mulher será informada sobre os centros de atendimentos, delegacias, } \\
\text { hospitais ou recursos disponíveis durante a pandemia. A mulher } \\
\text { também receberá, se necessário, um código promocional para solicitar } \\
\text { uma viagem de forma gratuita no app Uber e poder se deslocar. }\end{array}$ \\
\hline Aplicativo Magazine Luiza & $\begin{array}{l}\text { Botão inserido no aplicativo da empresa Magazine Luiza, que liga } \\
\text { diretamente ao } 180 \text { para denunciar violência doméstica. }\end{array}$ \\
\hline Mapa do acolhimento & $\begin{array}{l}\text { Plataforma que conecta uma rede de advogadas e psicólogas voluntárias } \\
\text { às mulheres que sofrem ou sofreram violência de gênero. }\end{array}$ \\
\hline ISA.Bot & $\begin{array}{l}\text { Inteligência artificial criada pela organização Think Olga e o Mapa de } \\
\text { Acolhimento para o Messenger e Google Assistente. Ao enviar uma } \\
\text { mensagem no inbox da página ISA.bot ou pedir ao Google Assistente } \\
\text { para falar com Robô Isa, ele oferece orientações às mulheres vítimas de } \\
\text { violência doméstica. }\end{array}$ \\
\hline Aplicativo PenhaS & $\begin{array}{l}\text { Aplicativo que fornece informações sobre os direitos das mulheres, } \\
\text { mapa das delegacias e serviços de atendimento mais próximos, canal } \\
\text { para acolhimento e diálogo, além de um botão de pânico para acionar } \\
\text { pessoas de confiança e ativação de gravação de áudio para produção } \\
\text { de provas. }\end{array}$ \\
\hline Startup Mete a Colher & $\begin{array}{l}\text { Startup que utiliza da tecnologia para o combate à violência contra } \\
\text { mulheres. Reúne, através das suas mídias digitais, voluntárias para } \\
\text { acolhimento, orientações jurídicas e suporte para mulheres em situação } \\
\text { de violência encontrarem uma vaga no mercado de trabalho. }\end{array}$ \\
\hline
\end{tabular}

Embora as iniciativas mencionadas sejam extremamente importantes para o combate à VPI, todas requerem que a mulher em contexto de VPI acione esses mecanismos. Por isso, campanhas publicitárias são essenciais para a disseminação de informações, bem como orientações e incentivo à população para que ofereçam ajuda ao presenciarem uma 
mulher em situação de violência, por meio do acionamento de serviços da rede formal de proteção, anteriormente descritos, como o Ligue 180 (ONU Mulheres, 2020; Instituto Avon, 2020).

O Instituto Avon, em parceria com instituições da iniciativa privada e do setor público, criou o Programa Você Não Está Sozinha: um plano de ações para combate aos impactos do isolamento às mulheres, seja facilitando o acesso para pedidos de ajuda, seja mediando os recursos disponíveis às necessidades básicas e psicológicas de cada uma. Além desse acolhimento e apoio, o programa tem se preocupado em dar visibilidade ao problema da violência doméstica através de campanhas publicitárias, para que a sociedade civil também se mobilize em oferecer auxílio a mulheres vítimas de violência (Instituto Avon, 2020).

A Globo, a GNT e a ONU Mulheres igualmente se uniram para lançar uma campanha de prevenção e combate à violência doméstica no isolamento social veiculada na televisão, levando a mensagem da importância de que a população esteja atenta a quaisquer sinais de violência advindos dos lares vizinhos, para que interfiram denunciando ou oferecendo apoio às mulheres que estão nessa situação de vulnerabilidade (ONU, 2020). Outra campanha que chama a atenção da população aos sinais de violência em pessoas próximas foi a realizada pelo Instituto Maria da Penha, em conjunto com as agências de publicidade F.biz e Vetor Zero. Por intermédio de um vídeo, retratam uma mulher que entrou para a videochamada com os colegas de trabalho apresentando comportamento que causou estranheza em uma das mulheres presentes na chamada, a qual, ao investigar a situação e confirmar de que se tratava de um caso de VPI, aciona a polícia (F.biz., 2020). A campanha chama a atenção da população para que esteja atenta a tais sinais ou qualquer mudança de comportamento que possa observar e se disponha a apoiar a vítima.

\section{Considerações Finais}

Derivada de uma cultura patriarcal e de desigualdade de gênero, a violência contra a mulher constitui um grave problema de saúde pública. O presente artigo teve como foco a violência sofrida por mulheres especificamente pelo parceiro íntimo (VPI), ou seja, qualquer comportamento dentro de um relacionamento íntimo que causa problemas físicos, psicológicos ou sexuais às mulheres na relação.

As graves consequências físicas e psicológicas da VPI na vida das mulheres são inegáveis. Em consonância com a Plataforma de Ação de Pequim, a Lei Maria da Penha prevê a proteção das mulheres no Brasil, por meio da articulação de uma rede de proteção e enfrentamento da violência contra a mulher em todo o território nacional. Sabemos que a rota crítica para que essas mulheres saiam da situação de violência depende, em grande medida, da acessibilidade e efetividade dessas redes de apoio. Contudo, o distanciamento social obrigatório decorrente da pandemia de covid-19 tem intensificado a VPI, já tão presente na vida de uma parcela significativa de mulheres. O confinamento, as dificuldades econômicas e a vigilância constante do parceiro dificultam o acesso às redes de apoio formais e informais.

Diversas iniciativas que facilitam a denúncia e o fortalecimento das redes de apoio à muIher foram encorajadas nesse período e sistematizadas no presente artigo. Dentre as estratégias de combate à VPI desenvolvidas pela rede de apoio formal, destacam-se o papel do poder público por meio do desenvolvimento de plataformas digitais, aplicativos, sites e 
campanhas que favorecem a denúncia de casos de violência; e o papel dos profissionais de saúde, os quais demandam protocolos específicos e capacitação no atendimento às vítimas de VPI para identificação de sinais e encaminhamento de casos suspeitos, em integração com a rede de proteção à mulher. Quanto às redes de apoio informais, projetos e iniciativas foram desenvolvidos por coletivos e empresas para contribuir para a denúncia de situações de violência e acolhimento das vítimas, tais como aplicativos, cartilhas e inteligências artificiais, bem como campanhas de conscientização da população e combate à violência doméstica.

Considera-se como limitação das análises realizadas no presente artigo que, por tratar-se de uma pandemia sem precedentes, poucos estudos envolvendo a VPI e contextos de isolamento social foram encontrados. A ausência de dados acerca dos caminhos das denúncias, de que forma conectar as mulheres aos serviços de proteção e quanto à efetividade de tais iniciativas na promoção da proteção das mulheres somam-se como limitações.

A partir da presente análise, sugere-se: que iniciativas como as elencadas se mantenham ativas após a pandemia; que metodologias de pesquisa para coleta de dados durante a pandemia sejam elaboradas e estudos de avaliação da efetividade dessas iniciativas sejam desenvolvidos e disseminados; que os serviços de proteção à mulher sejam ampliados e adaptados de forma que o fluxo da rede se torne mais acessível, contínuo e efetivo. Do ponto de vista do poder público, espera-se que haja um maior investimento na formação e no apoio de profissionais de saúde e da rede de proteção à mulher, bem como na elaboração de campanhas publicitárias de conscientização do fenômeno da VPI, de maneira a viabilizar o início da rota crítica dessas mulheres vítimas de violência.

Embora a Lei Maria da Penha preveja a assistência e proteção às mulheres vítimas de violência em todo o país, a prevalência da violência contra a mulher, que já era alta e subnotificada antes, mostra-se ainda mais preocupante em tempos de isolamento social. Iniciativas como as descritas no presente artigo contribuem para o acolhimento e a denúncia dos casos de VPI e reforçam a necessidade da conscientização, do fortalecimento e da articulação da rede de proteção à mulher, de forma a promover a proteção efetiva dessas mulheres e permitir que consigam usufruir do seu direito de viverem livres de violência.

\section{Referências}

Albuquerque Netto, L., Moura, M. A. V., Queiroz, A. B. A., Leite, F. M. C., \& Silva, G. F. (2017). Isolamento de mulheres em situação de violência pelo parceiro íntimo: Uma condição em redes sociais. Escola Anna Nery, 21(1), e20170007.

Almeida, L. R., Silva, A. T. M. C., \& Machado, L. S. (2013). Jogos para capacitação de profissionais de saúde na atenção à violência de gênero. Revista Brasileira de Educação Médica, 37(1), 110-119. doi:https://www.doi.org/10.1590/S0100-55022013000100016 Aun, H. (2020). Projeto oferece apoio gratuito a vítimas de violência doméstica na quarenta. Catraca Livre. Recuperado de https://catracalivre.com.br/cidadania/projeto-acolhevitimas-de-violencia-domestica-na-quarentena/

Baragatti, D. Y., Carlos, D. M., Leitão, M. N. C. da, Ferriani, M. G. C. das, \& Silva, E. M. (2018). Critical path of women in situations of intimate partner violence. Revista Latino-Americana de Enfermagem, 26, e3025. doi:https://www.doi.org/10.1590/1518-8345.2414.3025

Borges, B. (2020). Projeto 'Justiceiras' recebe denúncias de violência contra mulher durante quarentena. Agência Patrícia Galvão. Recuperado de https://agenciapatriciagalvao.org. 
$\mathrm{br} /$ violencia/projeto-justiceiras-recebe-denuncias-de-violencia-contra-mulher-durantequarentena-sp-concentra-casos/

Brasil. (2006). Lei n. 11.340 (7 de agosto). Cria mecanismos para coibir a violência doméstica e familiar contra a mulher. Brasília, DF. Recuperado de http://www.planalto.gov.br/ ccivil_03/_ato2004-2006/2006/lei/l11340.htm

Brasil. (2003). Lei n. 10.778 (24 de novembro). Estabelece a notificação compulsória, no território nacional, do caso de violência contra a mulher que for atendida em serviços de saúde públicos ou privados. Brasília, DF. Recuperado de http://www.planalto.gov.br/ ccivil_03/LEIS/2003/L10.778.htm

Brasil. Ministério da Mulher, da Família e dos Direitos Humanos. (2011). Pacto Nacional pelo Enfrentamento à Violência contra as Mulheres. Brasília, DF: Secretaria de Políticas para as Mulheres da Presidência da República.

Campos, C. H. (2015). Desafios na implementação da Lei Maria da Penha. Revista Direito GV, 11(2), 391-406. doi: https://www.doi.org/10.1590/1808-2432201517

Cavalcanti, G. M. B., Amorim A. V. B., Queiroz G. S., Cruz N. M., Costa R. L., \& Bezerra K. F. O. (2020). A violência contra a mulher no sistema único de saúde. Revista Online de Pesquisa, 12(1), 146-154. doi:https://www.doi.org/10.9789/2175-5361.rpcfo.v12.7148

Cerioni, C. (2020). Rappi cria botão contra violência doméstica em ação com advogadas e psicólogas. Exame. Recuperado de https://exame.com/pme/ rappi-cria-botao-de-violencia-domestica-em-acao-com-advogadas-e-psicologas/

Defensoria Pública do Estado de São Paulo. (2020). Guia rápido Direito das Mulheres e COVID-19. São Paulo: Núcleo Especializado de Promoção e Defesa dos Direitos das Mulheres.

Departamento de Informática do Sistema Único de Saúde. (2019). Dados extraídos da categoria Violência doméstica, sexuale/ou outras violências [Dados de saúde]. Recuperado de http://tabnet.datasus.gov.br/cgi/deftohtm.exe?sinannet/cnv/violebr.def

D'Oliveira, A. F. P. L., \& Schraiber, L. B. (2013). Mulheres em situação de violência: Entre rotas críticas e redes intersetoriais de atenção. Revista de Medicina, 92(2), 134-140. doi: https://www.doi.org/10.11606/issn.1679-9836.v92i2p134-140/

Dutra, M. L., Prates, P. L., Nakamura, E., \& Villela, W. V. (2013). A configuração da rede social de mulheres em situação de violência doméstica. Ciência \& Saúde Coletiva, 18(5), 1293-1304.

Entidade das Nações Unidas para a Igualdade de Gênero e o Empoderamento das Mulheres. (2020). Violência contra as mulheres e meninas é pandemia invisível, afirma diretora executiva da ONU Mulheres. ONU Mulheres, 7 abr. Recuperado de http://www. onumulheres.org.br/noticias/violencia-contra-as-mulheres-e-meninas-e-pandemia-dassombras-afirma-diretora-executiva-da-onu-mulheres/

F.biz. (2020). F.biz cria filme para o IMP que alerta sobre violência doméstica na quarentena. F.biz, 12 maio. Recuperado de https://www.fbiz.com.br/sala-de-imprensa/release/ fbiz-cria-filme-para-o-imp-que-alerta-sobre-violencia-domestica-na-quarentena

Fórum Brasileiro de Segurança Pública. (2020). Violência doméstica durante a pandemia de COVID-19 (Nota Técnica). Recuperado de https://site.cfp.org.br/wp-content/ uploads/2020/04/violencia-domestica-covid-19-v3.pdf 
Frossard, H. (2006). Instrumentos Internacionais de Direitos das Mulheres (Série Documentos). Ministério da Mulher, da Família e dos Direitos Humanos. Brasília, DF: Secretaria Especial de Políticas para as Mulheres.

Garbin, C. A. S., Garbin A. J. I., Dossi A. P., \& Dossi M. O. (2006). Violência doméstica: Análise das lesões em mulheres. Cadernos Saúde Pública, 22(12), 2567-2573. doi:https://www. doi.org/10.1590/S0102-311X2006001200007

García-Moreno, C., Hegarty, K., D’Oliveira, A. F. L., Kaziol-MacLain, J., Colombini, M., \& Feder, G. (2014). The health-systems response to violence against women. Lancet Online, 385(9977), 1567-1579. doi:https://www.doi.org/10.1016/S0140-6736(14)61837-7

Gregory, A., Taylor, A. K., Pitt, K., Feder, G., \& Williamson, E. (2019). “. . . The Forgotten Heroes": A Qualitative Study Exploring How Friends and Family Members of DV Survivors Use Domestic Violence Helplines. Journal Of Interpersonal Violence, 27(1). doi:https:// www.doi.org/10.1177/0886260519888199

Hasse, M., \& Vieira, E. M. (2014). Como os profissionais de saúde atendem mulheres em situação de violência? Uma análise triangulada de dados. Saúde em Debate, 38(102), 482-493. doi:https://www.doi.org/10.5935/0103-1104.20140045

Heise, L. L. (1998). Violence Against Women: An Integrated, Ecological Framework. Violence Against Women, 4(3), 262-290. doi:https://www.doi.org/10.1177/10778012980040030 02

Heise L. L. (2011). What works to prevent partner violence? An evidence overview. London: Strive Research Consortium.

Instituto Avon. (2020). Programa Você Não Está Sozinha. Avon. Recuperado de https://www. avon.com.br/instituto-avon/isoladassimsozinhasnao\#

Instituto Nelson Wilians. (2020). Movimento protege mulheres da violência em meio à COVID-19. INW. Recuperado de https://inw.org.br/violencia-contra-mulher-covid-19/

Instituto de Segurança Pública do Rio de Janeiro. (2021). Monitor da violência doméstica e familiar contra a mulher no período de isolamento social. ISP. Recuperado de http:// www.ispvisualizacao.rj.gov.br/monitor/index.html

Johns Hopkins University. (Dashboard). (2020). COVID-19 Dashboard by the Center for Systems Science and Engineering (CSSE) at Johns Hopkins University (JHU). Recuperado de https://coronavirus.jhu.edu/map.html

Kind, L., Orsini, M. L., Nepomuceno, V., Gonçalves, L., Souza, G. A., \& Ferreira, M. F. F. (2013). Subnotificação e (in)visibilidade da violência contra mulheres na atenção primária à saúde. Cadernos de Saúde Pública, 29(9), 1805-1815. doi:https://www.doi. org/10.1590/0102-311X00096312

Krug, E. G., Mercy, J. A., Dahlberg, L. L, \& Zwi, A. B. (2002). The world report on violence and health. The Lancet, 360(9339), 1083-1088, doi:https://www.doi.org/10.1016/ s0140-6736(02)11133-0

Mapa do acolhimento. (2020). Nenhuma mulher deve sofrer sozinha. Mapa do Acolhimento. Recuperado de https://www.mapadoacolhimento.org/\#block-9884

Office for National Statistics. (2016). Intimate Personal Violence and Partner Abuse. ONS, 11 fev. Recuperado de https://www.ons.gov.uk/peoplepopulationandcommunity/crimeandjustice/ compendium/focusonviolentcrimeandsexualoffences/yearendingmarch2015/chapter4inti matepersonalviolenceandpartnerabuse\#sources-of-support-for-partner-abuse-victims 
Organização das Nações Unidas. (2020). Todos na luta contra uma pandemia sem precedentes. ONU, 3 abr. Recuperado de https://news.un.org/pt/story/2020/04/1709332

Osis, M. J. D, Duarte, G. A., \& Faúndes, A. (2012). Violência entre usuárias de unidades de saúde: prevalência, perspectiva e conduta de gestores e profissionais. Revista de Saúde Pública, 46(2), 351-358. doi:https://www.doi.org/10.1590/S0034-89102012005000019

Peterman, A., Potts, A., O’Donnell, M., Thompson, K., Shah, N., Oertelt-Prigione, S., Gelder, N. (2020). Pandemics and Violence Against Women and Children (Working Paper, 528). Washington, DC: Center For Global Development.

Sagot, M. (2000). Ruta crítica de las mujeres afectadas por la violencia intrafamiliar en América Latina: Estudios de caso de diez países. San José: Organización Panamericana de la Salud.

Schraiber, L. B., \& D'Oliveira, A. F. P. L. (2002). Violence against women and Brazilian health care policies: A proposal for integrated care in primary care services. International Journal of Gynecology and Obstetrics, 78(suppl. 1), S21-S25. doi:https://www.doi.org/10.1016/ S0020-7292(02)00040-1

Sinclair, D. (2019). Por que a mulher permanece em um relacionamento violento? (M. Bonomi \& L. C. A Williams, Trad.). In L. C. A. Williams, J.M.D. Maia, K.A. Rios, \& S.M D’Affonseca (Orgs.), Aspectos psicológicos da violência (Vol. 1, pp. 51-58). Curitiba: Juruá.

Soares, B. M. (2005). Enfrentando a violência contra a mulher: Orientações práticas para profissionais e voluntários (as). Brasília, DF: Secretaria Especial de Políticas para as Mulheres. Recuperado de https://www12.senado.leg.br/institucional/omv/entendaa-violencia/pdfs/enfrentando-a-violencia-contra-a-mulher-orientacoes-praticas-paraprofissionais-e-voluntarios

Uber. (2020). Instituto Avon, Uber e Wieden+Kennedy lançam ferramenta para ajudar mulheres vítimas de violência doméstica. Uber, 28 abr. Recuperado de https://www.uber. $\mathrm{com} / \mathrm{pt}$-BR/newsroom/instituto-avon-uber-e-wiedenkennedy-lancam-ferramenta-paraajudar-mulheres-vitimas-de-violencia-domestica/

World Health Organization. (2014). Global status report on violence prevention. Genebra: WHO. Recuperado de https://www.who.int/publications/i/item/9789241564793

World Health Organization. (2016). Global plan of action: Health systems address violence against women and girls. Genebra: WHO. Recuperado de https://apps.who.int/iris/ handle/10665/251664

World Health Organization. (2020). Coronavirus disease (COVID-19): Violence against women. WHO, 15 abr. Recuperado de https://www.who.int/emergencies/ diseases/novel-coronavirus-2019/question-and-answers-hub/q-a-detail/ violence-against-women-during-covid-19

Vieira, P. R., Garcia, L. P., \& Maciel, E. L. N. (2020). Isolamento social e o aumento da violência doméstica: O que isso nos revela? Revista Brasileira de Epidemiologia, 23(e200033). doi:https://www.doi.org/10.1590/1980-549720200033

\section{Sobre as autoras:}

Luciana Barbalho Pontes: Doutora em Psicologia pela Universidade Federal de São Carlos (UFSCar). Mestre em Psicologia pela UFSCar. Graduada em Psicologia pela Universidade Federal do Pará 
(UFPA). Atualmente é professora substituta na Universidade do Estado do Pará (UEPA). E-mail: lu_barbalho@yahoo.com.br, Orcid: https://orcid.org/0000-0002-8234-9729

Maria Beatriz Reis Dionísio: Mestranda em Psicologia pela Universidade Federal de São Carlos (UFSCar). Graduada em Psicologia pela UFSCar. Atualmente é pesquisadora do Laboratório de Análise e Prevenção à Violência (LAPREV/UFSCar). E-mail: beatrizreisdionisio@gmail.com, Orcid: https://orcid.org/0000-0001-5711-5237

Maria Alice Centanin Bertho: Mestre em Psicologia pela Universidade Federal de São Carlos (UFSCar). Graduada em Psicologia pela UFSCar. Atualmente é pesquisadora do Laboratório de Análise e Prevenção à Violência (LAPREV/UFSCar) E-mail: marialicebertho@gmail.com, Orcid: https://orcid.org/0000-0002-8205-4031

Viviane Dutra Gama: Mestre em Psicologia pela Universidade Federal de São Carlos (UFSCar). Graduada em Psicologia pela UFSCar. Atualmente é pesquisadora do Laboratório de Análise e Prevenção à Violência (LAPREV/UFSCar) e psicóloga do Núcleo de Atendimento e Formação em Psicologia Clínica e Jurídica. E-mail: viviane_dutrag@hotmail.com, Orcid: https://orcid.org/0000-0003-3180-6640

Sabrina Mazo D’Affonseca: Doutora em Psicologia pela Universidade Federal de São Carlos (UFSCar). Mestre em Educação Especial pela UFSCar. Especialista em Psicopedagogia pelo Centro Universitário Central Paulista (UNICEP). Graduada em Psicologia pela UFSCar. Atualmente é professora do Departamento de Psicologia e do Programa de Pós-Graduação em Psicologia da UFSCar e pesquisadora do Laboratório de Análise e Prevenção à Violência (LAPREV). E-mail: samazo@ufscar.br, Orcid: https://orcid.org/0000-0001-9103-0616

Recebido em: 03/07/2020 Última revisão: 05/05/2021

Aceite final: 17/05/2021 
